\title{
QCD analysis of the diffractive structure function $F_{2}^{D(3)}$
}

\begin{abstract}
C. Royon*, L. Schoeffel ${ }^{\dagger}$, J.Bartels ${ }^{\ddagger}$, H.Jung ${ }^{\S}$, R.Peschanski ${ }^{* *}$
The proton diffractive structure function $F_{2}^{D(3)}$ measured in the $\mathrm{H} 1$ and ZEUS experiments at HERA is analyzed in terms of both Regge phenomenology and perturbative QCD evolution. A new method determines the values of the Regge intercepts in "hard" diffraction, confirming a higher value of the Pomeron intercept than for soft physics. The data are well described by a QCD analysis in which point-like parton distributions, evolving according to the DGLAP equations, are assigned to the leading and sub-leading Regge exchanges. The gluon distributions are found to be quite different for H1 and ZEUS. A global fit analysis, where a higher twist component is taken from models, allows us to use data in the whole available range in diffractive mass and gives a stable answer for the leading twist contribution. We give sets of quark and gluon parton distributions for the Pomeron, and predictions for the charm and the longitudinal proton diffractive structure function from the QCD fit. An extrapolation to the Tevatron range is compared with CDF data on single diffraction. Conclusions on factorization breaking depend critically whether H1 (strong violation) or ZEUS (compatibility at low $\beta$ ) fits are taken into account.
\end{abstract}

\section{INTRODUCTION}

It is now experimentally well established at HERA $[1,2]$ that a substantial fraction of $e p$ events is contributable to diffraction, i.e. color singlet exchange, initiated by a highly virtual photon. Starting with the pioneering theoretical work of Ref. [3], the idea of a point-like structure of the Pomeron exchange opens the way to the determination of its parton (quark and gluon) distributions, where the Pomeron point-like structure can be treated in a similar way as (and compared to) the proton one. Indeed, leading twist contributions to the proton diffractive structure functions can be defined by factorization properties [4] in much the same way as for the full proton structure functions themselves. As such, they should obey DGLAP evolution equations [5], and thus allow for perturbative predictions of their $Q^{2}$ evolution.

Indeed, such parton distributions are very useful to investigate the difficult and longstanding problem of the nature of the Pomeron Regge singularity. On a phenomenological ground, they are the basis of MC simulations like RAPGAP [6] and they give a comparison basis with "hard" diffraction at Tevatron, where the factorization properties are not expected to be valid. Moreover, the study of diffractive parton distributions is also a challenge for the discussion of different approaches and models where other than leading twist contributions can be present in hard diffraction. Indeed, there exists strong presumptive evidence that higher twist effects may be quite important in diffractive processes contrary to non-diffractive ones which do not require (at least at not too small $Q^{2}$ ) such contributions. In fact, there are models which incorporate non-negligible contributions from higher twist components, especially for relatively small masses of the diffractive system. One of our goals is to take into account this peculiarity of diffractive processes.

The purpose of this paper is to derive parton distributions of the Pomeron from QCD fits of diffractive DIS crosssections determined at HERA [1,2]. By comparison with previous determinations contained in the experimental papers, we introduce some new techniques of parametrizations and discuss various points which have appeared in the domain:

-the precise determination of the effective Pomeron and Reggeon trajectories in Regge fits

-the determination of the parton distributions at Next-to-Leading Order (NLO) in the Pomeron comparing two methods, either using cuts in order to damp the expected higher twist contributions or by a global fit including a higher twist contribution taken from models

-the differences between parton distributions derived from ZEUS and H1 data

\footnotetext{
*Service de Physique des Particules, CE-Saclay, F-91191 Gif-sur-Yvette Cedex, France; Brookhaven National Laboratory, Upton, New York, 11973; University of Texas, Arlington, Texas, 76019

${ }^{\dagger}$ Service de Physique des Particules, CE-Saclay, F-91191 Gif-sur-Yvette Cedex, France

${ }^{\ddagger}$ II. Institut für Theoretische Physik, Universität Hamburg, Luruper Chaussee 149, D-22761 Hamburg

${ }^{\S}$ University of Lund, Soelvegatan 14, 22362 Lund, Sweden

** Service de Physique Théorique, CE-Saclay, F-91191 Gif-sur-Yvette Cedex, France
} 
-the need to evaluate precisely the gluon content of the Pomeron and its error since it has strong impact on the discussion of diffractive charm and longitudinal diffractive structure functions

-the extrapolation of the diffractive structure function to single diffraction in $p \bar{p}$ collisions at Tevatron could give a precise evaluation of the factorization breaking.

Our paper is structured in the following way: In section II, we show Regge fits and the corresponding determination of intercepts from H1 (II-A) and ZEUS (II-B) data using a new method described in the appendix A1. In section III we use the same data but with additional cuts including a high $\beta$ cut to determine the parton distributions of the Pomeron in a NLO DGLAP evolution framework. The possibility of two different fits for H1 (III-A) and a remaining difference between H1 and ZEUS (III-B) are thoroughly examined. In section IV, we perform a new global fit analysis by removing the high $\beta$ cut, and using models to provide the higher twist contribution. In IV-A we check the stability of the determination of the leading twist parton distributions of the Pomeron. In IV-B we discuss the obtained gluon density in the Pomeron (for H1 and ZEUS fits) and examine the corresponding predictions for the diffractive charm (IV-C) and longitudinal (IV-D) components. The final selection of parameters for H1 and ZEUS fits is given in appendix A2. In section $\mathbf{V}$ we discuss the important consequence of these determinations for single diffraction at the Tevatron, as measured by the CDF collaboration [7]. We see that the conclusions on factorization breaking are markedly different for the H1 and ZEUS determinations. Conclusions and outlook are presented in the final section VI, as a discussion of the introductory points.

\section{REGGE FITS AND DETERMINATION OF INTERCEPTS}

\section{A. Determination of the Pomeron and Reggeon intercepts from H1 data}

The diffractive structure function $F_{2}^{D(3)}$, measured from DIS events with large rapidity gaps, can be investigated in the framework of Regge phenomenology and expressed as a sum of two factorized contributions corresponding to a Pomeron and secondary Reggeon trajectories.

$$
F_{2}^{D(3)}\left(Q^{2}, \beta, x_{\mathbb{P}}\right)=f_{\mathbb{P} / \mathrm{p}}\left(x_{\mathbb{P}}\right) F_{2}^{\mathbb{P}}\left(Q^{2}, \beta\right)+f_{\mathbb{R} / \mathrm{p}}\left(x_{\mathbb{P}}\right) F_{2}^{\mathbb{R}}\left(Q^{2}, \beta\right) .
$$

In this parameterisation, $F_{2}^{\mathbb{P}}$ can be interpreted as the Pomeron structure function and $F_{2}^{\mathbb{R}}$ as an effective Reggeon structure function, with the restriction that it takes into account various secondary Regge contributions which can hardly be separated. The Pomeron and Reggeon fluxes are assumed to follow a Regge behaviour with linear trajectories $\alpha_{\mathbb{P}, \mathbb{R}}(t)=\alpha_{\mathbb{P}, \mathbb{R}}(0)+\alpha_{\mathbb{P}, \mathbb{R}}^{\prime} t$, such that

$$
f_{\mathbb{P} / p, \mathbb{R} / p}\left(x_{\mathbb{P}}\right)=\int_{t_{c u t}}^{t_{\text {min }}} \frac{e^{B_{\mathbb{P}, \mathbb{R}} t}}{x_{\mathbb{P}}^{2 \alpha_{\mathbb{P}, \mathbb{R}}(t)-1}} \mathrm{~d} t
$$

where $\left|t_{\text {min }}\right|$ is the minimum kinematically allowed value of $|t|$ and $t_{c u t}=-1 \mathrm{GeV}^{2}$ is the limit of the measurement.

In the first step we determine the value of the Pomeron and Reggeon intercepts $\left(\alpha_{\mathbb{P}, \mathbb{R}}(0)\right)$ from phenomenological fits of $\mathrm{H} 1$ data. In equation (1), the values of $F_{2}^{\mathbb{P}, \mathbb{R}}$ are treated as free parameters at each $\beta$ and $Q^{2}$ point. The values of $\alpha_{\mathbb{P}, \mathbb{R}}(0)$ are free parameters while $B_{\mathbb{P}, \mathbb{R}}$ and $\alpha_{\mathbb{P}, \mathbb{R}}^{\prime}$ are taken from hadron-hadron data $\left(\alpha_{\mathbb{P}}^{\prime}=0.26 \mathrm{GeV}^{-2}\right.$, $\alpha_{\mathbb{R}}^{\prime}=0.90 \mathrm{GeV}^{-2}, B_{\mathbb{P}}=4.6 \mathrm{GeV}^{-2}, B_{\mathbb{R}}=2.0 \mathrm{GeV}^{-2}$ ), since the data are not precise enough to determine the $t$-slopes [1]. In order to avoid the region that may be most strongly affected by a non-zero value of the ratio $R$ of the longitudinal to the transverse cross-sections, only data with $y<0.45$ are included in the fit.

Note that the fit with a single trajectory does not give a good description of the data $\left(\chi^{2} /\right.$ dof $=65.5 / 19$ with statistical errors only). This confirms the observations $[1,8]$ that secondary trajectories in addition to the Pomeron are required to describe diffractive $e p$ data. A much better fit is thus obtained when both a leading $(\mathbb{P})$ and a sub-leading $(\mathbb{R})$ trajectory are considered.

Since the sub-leading exchange is poorly constrained by the data, a second fit is performed to the H1 data in which the values of $F_{2}^{\mathbb{R}}\left(Q^{2}, \beta\right)$ are taken from a parameterisation of the pion structure function [9], with a single free normalization. We use the pion structure function of Ref. [9] to be able to extrapolate ${ }^{1}$ our fits to lower $Q^{2}$. In principle, an interference term could be introduced in equation (1), but this does not modify the $\chi^{2}$ of the fit.

\footnotetext{
${ }^{1}$ Note that the Duke Owens parametrisation [10] used in Ref. [1] is not valid at low $Q^{2}$, as it has a constant value for a $Q^{2}$ less than $4 \mathrm{GeV}^{2}$.
} 
The resulting value of $\alpha_{\mathbb{P}}(0)=1.20 \pm 0.09$ is in agreement with that obtained in Ref. [1] and is significantly larger than values extracted from soft hadronic data $\left(\alpha_{\mathbb{P}} \sim 1.08\right)$. Also, we find $\alpha_{\mathbb{R}}(0)=0.62 \pm 0.03^{2}$. The use of the pion structure function in our treatment is questionable as the Reggeon intercept is close to the rho value and not to the pion one. However, the $\mathrm{H} 1$ data where a proton or a neutron is tagged in the final state can be described nicely by pion and Reggeon exchanges where the Reggeon structure function is also taken to be the pion one [12]. We thus do not expect a significant bias due to this assumption.

A well-known technical problem for the determination of the Regge fit at each $\beta$ and $Q^{2}$ point is the correct identification of the actually free parameters. We use a new method described in appendix $\mathbf{A 1}$ and perform a direct fit of $\alpha_{\mathbb{P}}(0)$ and $\alpha_{\mathbb{R}}(0)$. Indeed, this method is designed to take into account the decorrelations between the parameters and to give a more reliable minimization procedure. Without using this method, many local minima are present in the $\chi^{2}$ which might give an incorrect value of the exponents.

This method leads to better and faster convergence properties for the Regge fits procedure when applied to H1 data since the number of parameters to be fitted is much smaller. We find the following values : $\alpha_{\mathbb{P}}(0)=1.20 \pm 0.02$ and $\alpha_{\mathbb{R}}(0)=0.62 \pm 0.02$ (statistical errors only), which are close to those determined previously. We keep these values fixed in the next sections.

\section{B. Determination of the Pomeron and Reggeon intercepts from ZEUS data}

We performed the same study using ZEUS data and we find a Pomeron intercept $\alpha_{\mathbb{P}}=1.13 \pm 0.04$, compatible with what has been obtained by the ZEUS collaboration [2]. This value is lower than the H1 value ${ }^{3}$ extracted in the previous section, however, the selection of the diffractive sample in ZEUS is different from H1 [2]. We will come back to this last point in the following. The Reggeon contribution is also found to be compatible with zero since the method of measurement (the so-called $M_{X}$ method) is not sensitive to the Reggeon component [2].

\section{EXTRACTION OF PARTON DISTRIBUTIONS IN THE POMERON}

\section{A. Parton distributions from $\mathrm{H} 1$ data}

As we have already indicated in Equation (1), the diffractive structure function $F_{2}^{D(3)}$ can be investigated in the framework of Regge phenomenology. Moreover, it has been suggested that the $Q^{2}$ evolution of these structure functions may be understood in terms of parton dynamics [3], i.e. as coming from leading twist, perturbative QCD contributions where parton densities are evolved according to DGLAP equations [5].

This idea has been tested in Ref. [1] with an iterative method using the CTEQ evolution code [13] over infinitesimal steps in $x$ space to solve the NLO DGLAP equations [5]. In the following we redo this analysis of H1 data with a new polynomial method to solve DGLAP equations at NLO [14].

We assign parton distribution functions to the Pomeron and to the Reggeon. A simple prescription is adopted in which the parton distributions of both the Pomeron and the Reggeon are parameterised in terms of non-perturbative input distributions at some low scale $Q_{0}^{2}=3 \mathrm{GeV}^{2}$.

As we said in the last chapter, the pion structure function [9] is assumed for the sub-leading Reggeon trajectory with a free global normalization to be determined by the data ${ }^{4}$.

For the Pomeron, a quark flavour singlet distribution $\left(z S_{q}\left(z, Q^{2}\right)=u+\bar{u}+d+\bar{d}+s+\bar{s}\right)$ and a gluon distribution $\left(z G\left(z, Q^{2}\right)\right)$ are parameterized in terms of coefficients $C_{j}^{(S)}$ and $C_{j}^{(G)}$ at $Q_{0}^{2}=3 \mathrm{GeV}^{2}$ as it was done in Ref. [1] such that

\footnotetext{
${ }^{2}$ Introducing interference terms reduces the value of the Reggeon exponent to 0.5, compatible with regge phenomenology. The fact we do not put any interference term leads to an effective value of the Reggeon exponent of 0.62 . On the other hand, the value of the Pomeron exponent remains constant with or without interference.

${ }^{3}$ This last value is more compatible with soft physics if one considers a global fit to all data from $\pi p, K p$ and $p p$ including the $1800 \mathrm{GeV} p \bar{p}$ cross-section [11].

${ }^{4}$ We checked that changing the pion structure function by some amount (20\%) does not change significantly the parton distributions.
} 


$$
\begin{aligned}
& z S\left(z, Q^{2}=Q_{0}^{2}\right)=\left[\sum_{j=1}^{n} C_{j}^{(S)} \cdot P_{j}(2 z-1)\right]^{2} \cdot e^{\frac{a}{z-1}} \\
& z G\left(z, Q^{2}=Q_{0}^{2}\right)=\left[\sum_{j=1}^{n} C_{j}^{(G)} \cdot P_{j}(2 z-1)\right]^{2} \cdot e^{\frac{a}{z-1}}
\end{aligned}
$$

where $z=x_{i / \mathbb{P}}$ is the fractional momentum of the Pomeron carried by the struck parton (in the following, we use indifferently $z$ or $\beta), P_{j}(\zeta)$ is the $j^{\text {th }}$ member in a set of Chebyshev polynomials, which are chosen such that $P_{1}=1$, $P_{2}=\zeta$ and $P_{j+1}(\zeta)=2 \zeta P_{j}(\zeta)-P_{j-1}(\zeta)$.

A sum of $n=3$ orthonormal polynomials is used so that the input distributions are free to adopt a large range of forms for a given number of parameters. Any bias towards a particular solution due to the choice of the functional form of the input distribution is therefore minimized.

The trajectory intercepts are fixed to $\alpha_{\mathbb{P}}=1.20$ and $\alpha_{\mathbb{R}}=0.62{ }^{5}$ (as explained in the last section). Only data points of $\mathrm{H} 1$ with $Q^{2} \geq 3 \mathrm{GeV}^{2}, \beta \leq 0.65, M_{X}>2 \mathrm{GeV}$ and $y \leq 0.45$ are included in the fit in order to avoid large higher twist effects and the region that may be most strongly affected by a non-zero value of $R$. We will discuss this point in the following when we take into account the higher twist contribution to the diffractive structure functions. The fits include 161 data points for 7 parameters ( 3 for the sea quark density, 3 for the gluon density and 1 for the normalization of the Reggeon contribution). Thus, we have 154 degrees of freedom.

To get solvable evolution equations, the parton distribution functions must approach zero as $z \rightarrow 1$. This is achieved by introducing the exponential term with a positive value of the parameter $a$. Unless otherwise indicated, in the following fits $a$ is set to 0.01 such that this term only influences the parametrisation in the region $z>0.9$. This term is only present to ensure the convergence and plays a role in a domain where we do not include data. (For instance, at $\beta=0.65$, its value is equal to 0.97.)

The functions $z S$ and $z G$ are evolved to higher $Q^{2}$ using the next-to-leading order DGLAP evolution equations. The contribution to $F_{2}^{\mathbb{P}}\left(\beta, Q^{2}\right)$ from charm quarks is calculated in the fixed flavour scheme using the photon-gluon fusion prescription given in [15]. The contribution from heavier quarks is neglected.

No momentum sum rule is imposed because of the theoretical uncertainty in specifying the normalization of the Pomeron or Reggeon fluxes and because it is not clear that such a sum rule is appropriate for the parton distributions of a virtual exchange. After fitting, however, we observed that the sum rules were fulfilled within $10 \%$ despite being not required in the fits.

The resulting parton densities of the Pomeron are presented in Fig. 1. One possible fit quoted here as fit 1 shows a large gluonic content. The quark contribution is much smaller compared to the gluon one. An other much less favoured fit, quoted here as fit 2, has a peaked gluon distribution at high $\beta^{6}$. It should be noticed that only the first solution is found if one imposes in the fit that the parton distributions have no zero over the full $\beta$ and $Q^{2}$ range, whereas the second fit appears when this condition is removed.

The appearance of a second solution in our fit to the H1 data is very reminiscent of the results presented in Ref. [16]. In fact, the general characteristics of the two solutions obtained in the present paper and of those found in Ref. [16] are quite similar: the difference in the gluon content at large $z$ (or $\beta$ ), and the instability of the high-gluon solution with respect to cuts in the data used for the fit. Nevertheless, one has to be careful in comparing the results of Ref. [16] with the present ones: whereas the former ones represent fits to the diffractive cross section $F_{2}^{D(3)}$, the present analysis describes results for diffractive parton densities.

The result of the fit ( fit 1) is presented in Fig. 2 together with the experimental values for H1 data points; we see on this figure the good agreement of the QCD fit with the data points, which supports the validity of the description of the Pomeron in terms of partons following QCD dynamics. In Fig. 3 we also compare the scaling violations obtained in the fit to the H1 measurement.

\footnotetext{
${ }^{5}$ If one fits the values of $\alpha_{\mathbb{P}}$ and $\alpha_{\mathbb{R}}$, one gets the same values as in Section II.

${ }^{6}$ Fit 2 shows a higher $\chi^{2}$ compared to fit 1 (the $\chi^{2}$ per degree of freedom is 1.15 for fit 1 and 1.25 for fit 2 ). Furthermore, fit 2 is quite unstable: changing the parameters a little modifies the gluon distribution at high $z$, and removing some data points at low or medium $\beta$ for instance destroys this fit completely. The quark distribution is similar for both fits, but the gluon distribution tends to be quite different at high values of $z$. This can be easily explained as no data above $z=0.65$ are included in the fits. Thus there is no constraint from the data at high $z$. Therefore, we keep fit 1 as the best fit for H1 diffractive cross-sections.
} 
Let us discuss the assumptions we made in the QCD fits. We took $Q_{0}^{2}=3 \mathrm{GeV}^{2}$ and we noticed that the $\chi^{2}$ of the fit increases significantly if we try to lower this value of $Q_{0}^{2}\left(\chi^{2} / \operatorname{dof}=1.5\right.$ if $\left.Q_{0}^{2}=1 \mathrm{GeV}^{2}\right)$, indicating that the fit cannot be extended to lower $Q^{2}$. We will come back to this point in the following.

We performed the fits including three Chebyshev polynomials. Including one more polynomial does not improve the $\chi^{2}$ of the fit, while two polynomials are also not enough to get a good convergence of the fit $\left(\chi^{2} /\right.$ dof $\left.=1.7\right)$.

The parametrizations used in this analysis are given in formulae (3) and (4): indeed, the behaviour at large $z$ is forced to go to 0 when $z$ goes to 1 by the term $\exp \left(\frac{a}{z-1}\right)$ which includes an essential singularity at $z=1$. We tried to perform the analysis with $a=0$ imposing the behaviour at large $z$ with a term in $(1-z)^{\alpha}$. With these assumptions, we did not get any fits with a good $\chi^{2}\left(\chi^{2} /\right.$ dof $\left.>2\right)$. The form of the parametrization for the parton distribution at large $z$ plays a non-negligible role in the fit procedure to ensure a quick convergence at high $z$. However, this term is large in a region where no data are included in the fit. On a more physical ground, it is interesting to note that this behaviour is in contrast with the one for proton (or even pion, cf. Drell-Yan analysis) structure functions for which the behaviour of the distribution tail is seen in a rather sizable region of $z$.

The positive scaling violations of diffractive structure functions lead to an important gluon content $z G(z)$ in the Pomeron (even at large $z$ ), which is what we get from QCD fits (see Fig. 3).

Moreover, with this gluon distribution, larger scaling violations at low $\beta$ are naturally associated with larger values of gluon splitting functions when $\beta$ gets lower. Now, it is also clear that, due to large measurement uncertainties, scaling violations and thus the determination of $\alpha_{s} z G$ are not very precise. The uncertainty on the value of $\alpha_{s}$ is negligible in this analysis, and we can get direct access to the uncertainty on the gluon density (determined from QCD fits). We get from the investigation of the scaling violations

$$
\frac{\delta G}{G} \simeq 25 \% .
$$

We believe that this (even not yet precise) error determination is a relevant piece of information, showing that the gluon density is not yet very well constrained.

In conclusion, the gluon content of the Pomeron is confirmed to be large (w.r.t. [1]). The flat gluon solution is favoured, and another result ( fit 2) appears if we allow that the parton distributions can possibly vanish in the kinematical range. Moreover this second solution is disfavoured due to its worse $\chi^{2}$ and instability and thus, we will not use this second solution in the following. The accuracy of the gluon determination is still quite poor. This provides a very nice perspective for diffractive analyses of more recent data where more accurate results will lead to a more precise QCD analysis. 

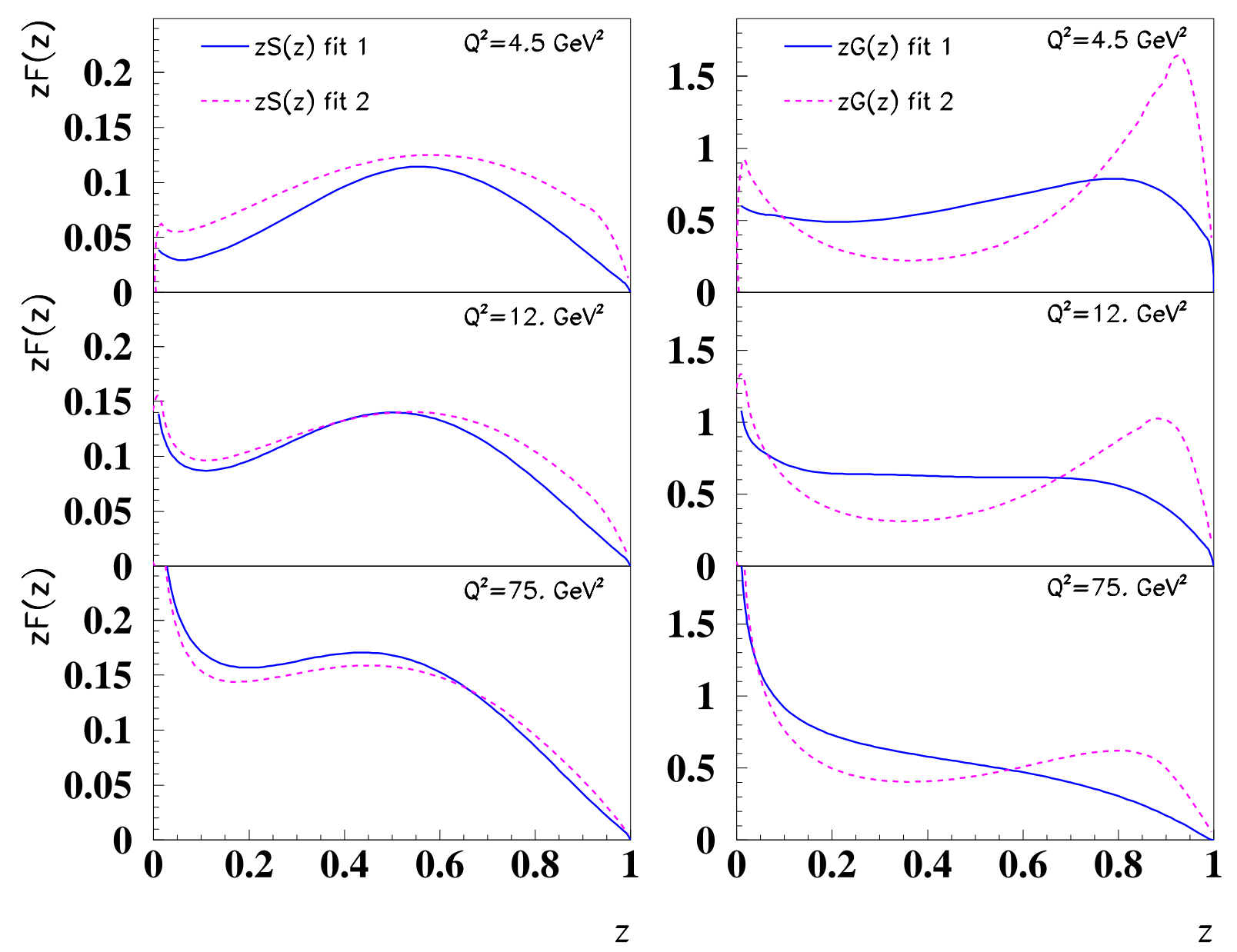

FIG. 1. Quark flavour singlet $(z S$, left) and gluon $(z G$, right) distributions of the Pomeron deduced as a function of $z$, the fractional momentum of the Pomeron carried by the struck parton, from the fit on $\mathrm{H} 1$ data points with $Q^{2} \geq 3 \mathrm{GeV}$. The solid (dashed) curve shows fit 1 ( fit 2) as discussed in the text $\left(\chi^{2} /\right.$ dof $=177.1 / 154=1.15$ for fit 1 and $\chi^{2} /$ dof $=192.5 / 154=1.25$ for fit 2 with statistical errors only). The parton densities are normalised to represent $x_{\mathbb{P}}$ times the true parton densities multiplied by the flux factor at $x_{\mathbb{P}}=0.005$ (this will always be the case in all the following figures). 


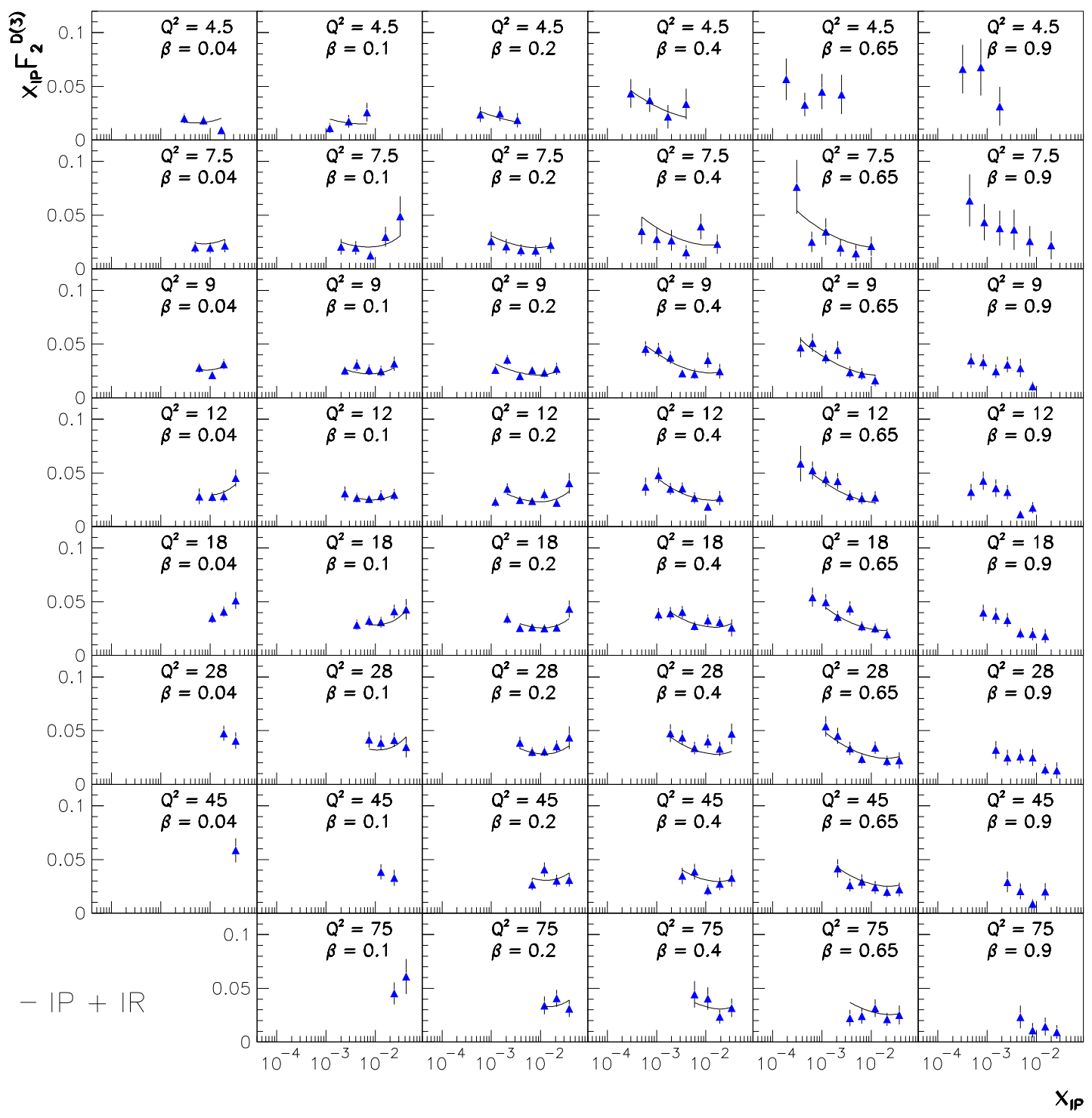

FIG. 2. The H1 data points for $x_{\mathbb{P}} F_{2}^{D(3)}$ are shown with the result of the QCD fit ( fit 1) described in the text; the result of the fit is shown only in bins included in the minimization procedure. 


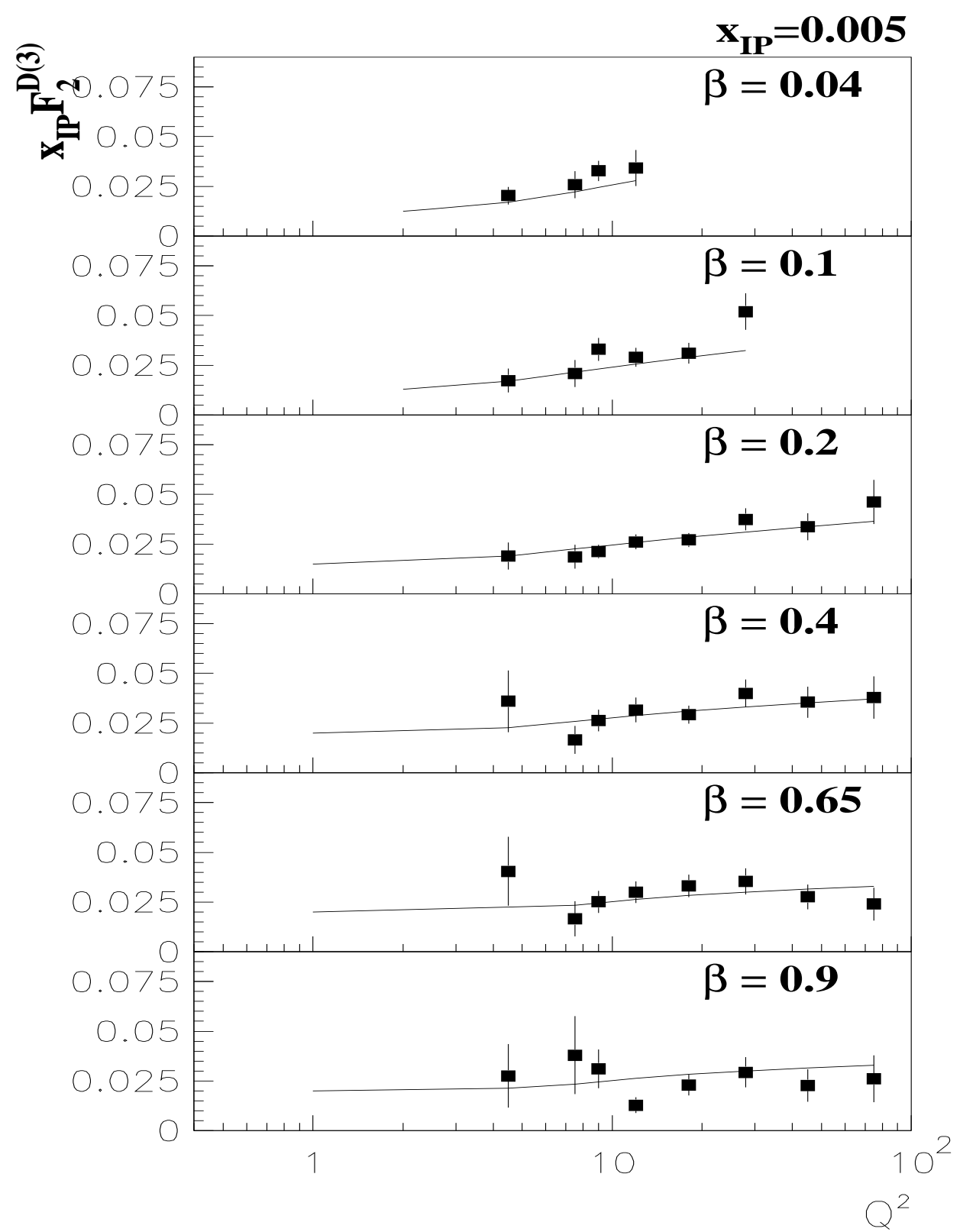

FIG. 3. The structure function $x_{\mathbb{P}} \cdot F_{2}^{D(3)}$ at $x_{\mathbb{P}}=0.005$, presented as a function of $Q^{2}$ in bins of $\beta$, over the full $Q^{2}$ range accessed with the H1 data. The superimposed line represents the result of the QCD fit to H1 data described in section IIIA.

\section{B. Parton distributions from ZEUS data}

We have redone this QCD analysis with ZEUS diffractive cross-section measurements [2] applying the same cuts. Note that for the ZEUS fits, there are only 6 parameters since the method of measurement is not sensitive to the Reggeon contribution [2]. The Pomeron intercept is fixed to $\alpha_{\mathbb{P}}=1.13$ as determined from Regge fits on ZEUS data $[2]$.

The resulting parton distributions are presented in Fig. 4 together with the H1 results. As the Pomeron intercepts are different for $\mathrm{H} 1$ and ZEUS data, the parton densities are normalized to represent $x_{\mathbb{P}}$ times the true parton densities multiplied by the flux factor at $x_{\mathbb{P}}=0.005$. 
We notice some large discrepancies, especially in the gluon density ${ }^{7}$. The gluon density derived from ZEUS measurements is lower that the $\mathrm{H} 1$ fit results. The data are compared directly in Fig. 6 where we rescaled the H1 data to the ZEUS bins (the rescaling is not very model-dependent since the shifts in $Q^{2}$ and $\beta$ between both experiments are quite small, and we performed the rescaling using models in Ref. [16] [17], which lead to the same result). In addition to this treatment of data, about $10 \%(30 \%)$ of the cross-section should be subtracted to take into account proton dissociation contribution in the H1 (ZEUS) cross-section measurement. From Fig. 6, we notice that the main differences are located in the highest $Q^{2}$ bins $\left(Q^{2} \sim 60 . \mathrm{GeV}^{2}\right)$ after this additional correction and could be due to the different methods used to do this measurement (rapidity gap method or so-called $M_{X}$ method $[1,2])$. However, there are only 30 ZEUS data points entering in the QCD analysis compared to 161 for H1 analysis, the experimental uncertainties being of the same order. Thus, the statistical power of the ZEUS fits is considerably lower. We can estimate the statistical uncertainty on the gluon distribution to be about $50 \%$ for ZEUS data (to be compared with $25 \%$ for $\mathrm{H} 1$ data). The differences in the quark densities are much smaller taking into account the fact that there is no ZEUS data at $Q^{2}=4.5 \mathrm{GeV}^{2}$ and low $\beta$. Differences in the parton distributions are clearly due to small differences in the data (see Fig. 4). We see differences in the $Q^{2}=60 \mathrm{GeV}^{2}, \beta=0.7$, or $Q^{2}=8 \mathrm{GeV}^{2}, \beta=0.2$ bins.

As the fit results are quite different for both experiments, we choose to make predictions for the longitudinal and charm structure functions for both sets of parameters.

\footnotetext{
${ }^{7}$ Note that such different gluon densities can lead to similar values of the structure functions $F_{2}^{D(3)}$ (see Fig. 4) because the diffractive structure function is more sensitive to the quark density (the photon couples directly to quark). This will however lead to differences in the charm structure function as it is much more sensitive to the gluon structure function.
} 


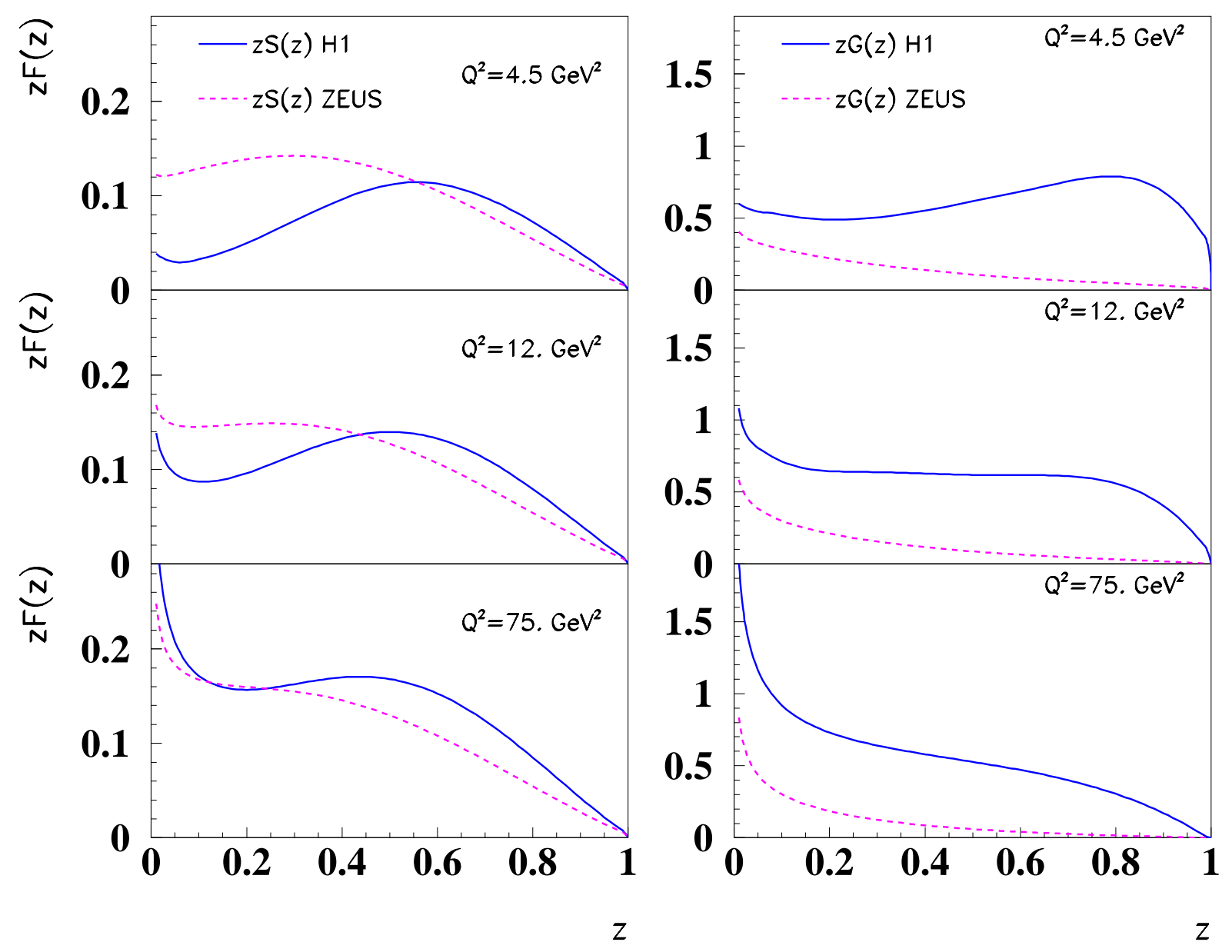

FIG. 4. Quark flavour singlet $(z S$, left) and gluon $(z G$, right) distributions of the Pomeron derived from H1 diffractive data $\left(\chi^{2} /\right.$ dof $=177.1 / 154=1.15$, full lines $)$ and ZEUS diffractive data $\left(\chi^{2} /\right.$ dof $=29.3 /(30-6)=1.22$, dashed lines $)$. 


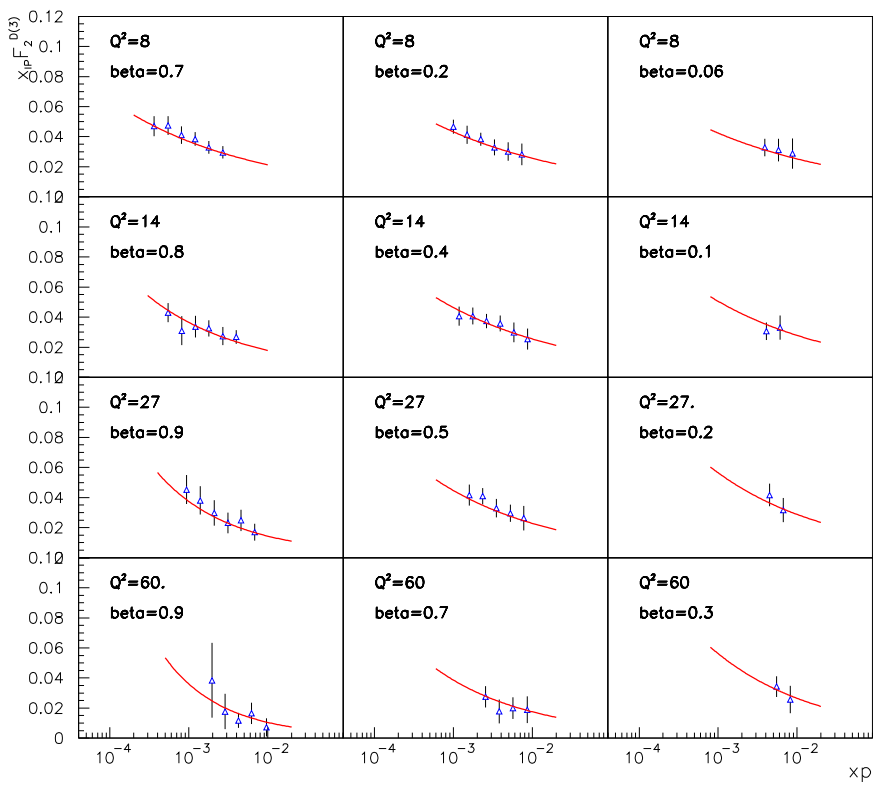

FIG. 5. The ZEUS data points on $x_{\mathbb{P}} F_{2}^{D(3)}$ are shown with the result of the QCD fit described in the text.

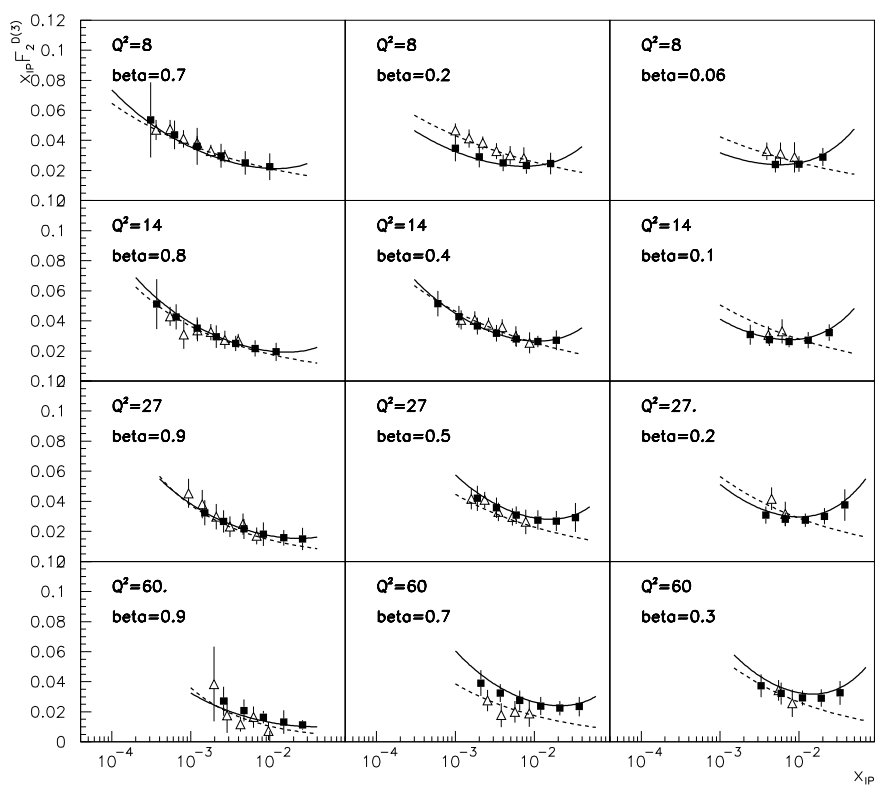

FIG. 6. Comparison of H1 and ZEUS data on $x_{\mathbb{P}} F_{2}^{D(3)}$ (respectively black squares and open triangles) with the result of the QCD fits: full line for the H1 QCD fit result and dashed line for ZEUS. Note that the H1 points have been rebinned to ZEUS $Q^{2}$ and $\beta$ values. 


\section{GLOBAL FITS}

\section{A. Higher-twists and global fits}

The H1 fit ( fit 1) is our reference and we attempt to refine it by extending the number of data points included into the fit procedure. The problem is that the cuts described above are essential to avoid the experimental domain where non-perturbative power contributions could be large and we cannot suppress these cuts without subtracting in another way these power contributions (or higher-twist terms). However, some parametrizations for higher-twist contributions have been proposed [16,17] after a dedicated analysis of H1 data.

We use the results of Ref. [16,17] to subtract the higher twist component from the $F_{2}^{D(3)}$ measurements at high $\beta$ and we redo the QCD analysis with all data points included, which gives 224 data points for H1.

The resulting parton distribution are presented in Fig. 7 for the fit to the H1 data using the higher twist determination of Ref. $[16]^{8}$. We notice the perfect agreement between parton distributions extracted with this procedure and with our former analysis described above. However, the statistical power of the new procedure is better due to the larger number of degrees of freedom. We also note that the $\chi^{2} /$ dof is smaller when we perform the fit after subtracting the higher twist component (1.05 compared to 1.15, see Fig. 7). This implies that a small residual higher twist component is still present near $\beta=0.65$ which influences the quality of the fit, and thus, it is quite dangerous to cut directly in the data.

As we now include all data in the fit, the assumption we made on the a parameter of Equation (4) may play a role. We checked as before that the convergence is not possible if we include a polynomial instead of an exponential form (the $\chi^{2}$ per degree of freedom increases up to 2.). However, the value of $a$ is not fundamental as another value $\left(a=10^{-3}\right)$ also leads to a good fit.

The parton distributions extracted with the global procedure are now our reference distributions. The parameters of the gluon and quark densities are given in appendix A2.

From the ZEUS data, we also get comparable results with or without higher twist corrections, leading to the same parton distributions as shown in Fig. 4.
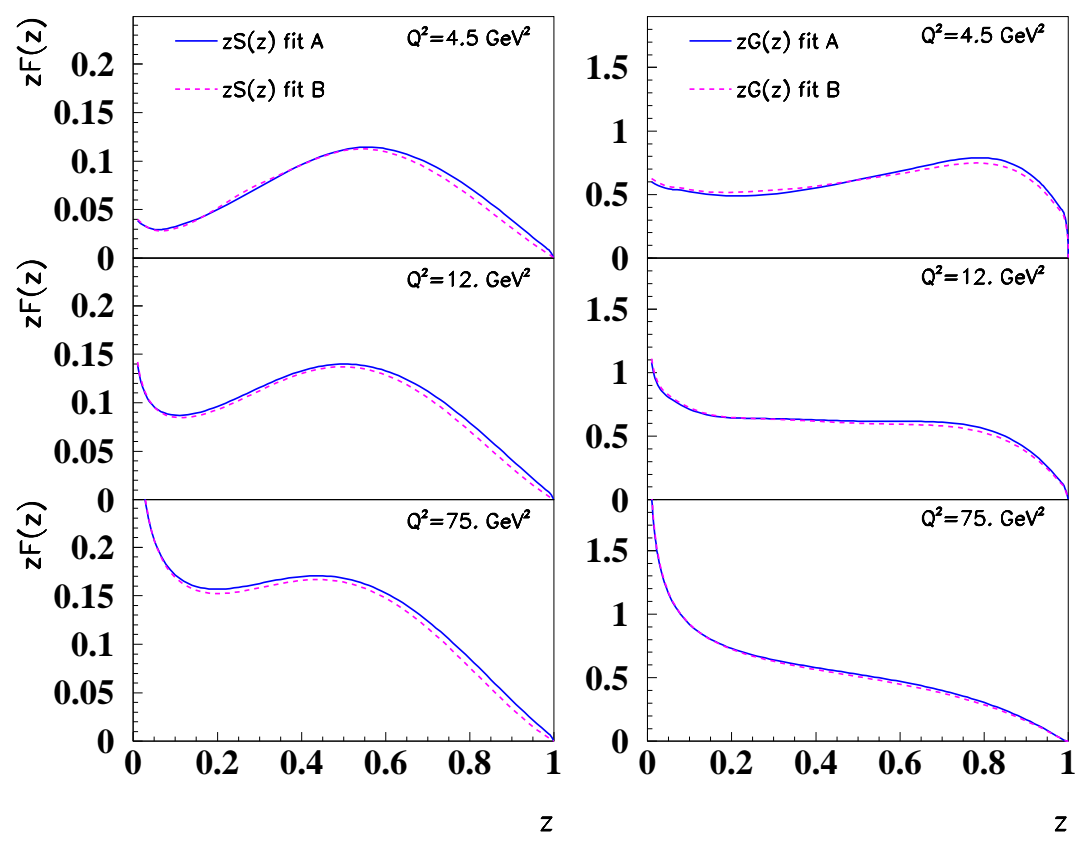

FIG. 7. Parton distributions extracted from H1 data with the standard QCD fits $\left(\chi^{2} /\right.$ dof $=177.1 / 154=1.15$, full lines, fit A) compared to distributions extracted from the global fit analysis using all H1 measurements $\left(\chi^{2} /\right.$ dof $=228.4 /(224-6)=1.05$, dashed lines, fit B).

\footnotetext{
${ }^{8}$ We checked that using another higher twist parametrisation from Ref. [17] which uses a BFKL-dipole type approach [18,19] does not change our results.
} 


\section{B. Comparison of the gluon density in the proton and in the Pomeron}

As can be seen in Fig. 8, the gluon density derived from our global QCD fits of H1 diffractive measurements is very different from the one derived from QCD fits of $F_{2}$ [24]. However, since the uncertainty of our determination is large $\left(\frac{\delta G}{G} \geq 25 \%\right.$ as we have mentioned above), one can wonder whether $F_{2}^{D(3)}$ could be described using the same gluon density as for the proton total structure function $F_{2}$.

We have taken the gluon density of Ref. [24] (we simply identify Bjorken- $x$ with $\beta$ from this parametrisation) and we have redone the QCD analysis of $F_{2}^{D(3)} \mathrm{H} 1$ data by fitting only the sea quark distribution using directly the gluon density inside the proton. As explained above we have also used the prescription of Ref. [16] to subtract the higher-twist contribution from diffractive measurements, which gives 224 data points and 3 parameters in the fit. This procedure leads to $\chi^{2} / d o f=304.2 /(224-3)=1.37$ which is worse than our reference fit for diffractive data $\left(\chi^{2} /\right.$ dof $\left.=228.4 /(224-6)=1.05\right)$. This result suggests that the gluon density of the Pomeron is quite different from the gluon density of the proton ${ }^{9}$. The difference is smaller between the result of the ZEUS fit and the gluon density inside the proton (in the case of the ZEUS fit, the gluon density in the Pomeron is found to be smaller than in the H1 case). Both H1 and ZEUS gluon densities in the Pomeron are however incompatible with the gluon in the proton at high $x$ or $\beta$.

The fit results compared to the data show large discrepencies at low $z$ in all $Q^{2}$ bins. It is impossible to describe diffractive data at low $\beta$ using the same gluon density as in the proton.

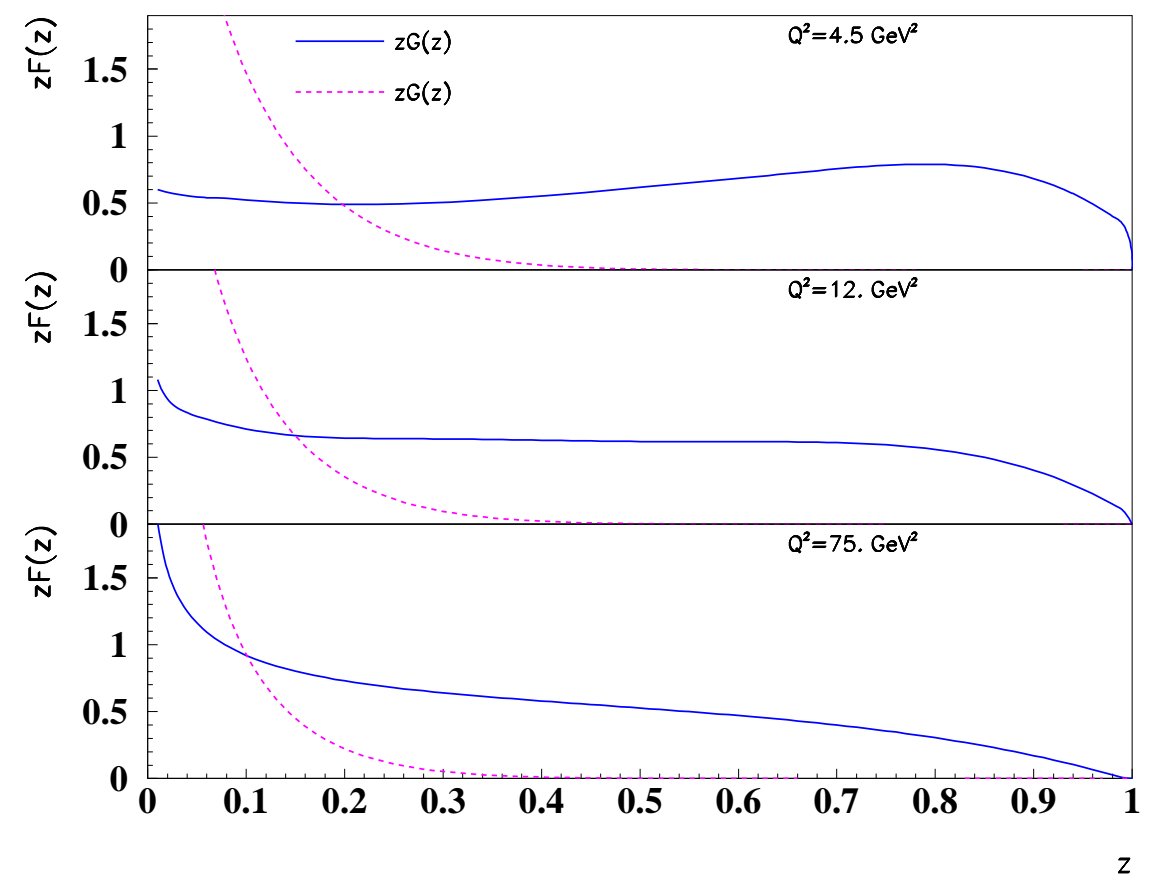

FIG. 8. Gluon density fitted from diffractive data (global fits, full lines) compared with the gluon density extracted from $F_{2}$ QCD fits (dashed lines).

\footnotetext{
${ }^{9}$ Note that we have also redone this procedure with a free normalization for the gluon distribution. However, this normalization is not changed during the fitting procedure. Thus we get the same results as before.
} 


\section{Fit extrapolations in $Q^{2}$}

We now extrapolate our QCD fits using H1 and ZEUS data to low and high $Q^{2}$ to look for a possible kinematical domain where the differences in the H1 and ZEUS parton distributions can lead to differences in $F_{2}^{D}$.

As we mentioned before in section IIIA, it is difficult to lower the starting scale $Q_{0}^{2}$ to get a prediction for $F_{2}^{D(3)}$ at low $Q^{2}$ because the quality of the fit is much worse. Thus, we perform a low $Q^{2}$ backward evolution using $Q_{0}^{2}=3$ $\mathrm{GeV}^{2}$. On the other hand, it has been recently noted by the H1 and ZEUS collaborations [20] that the H1 1995 and ZEUS 1995 preliminary data at low $Q^{2}$ cannot be described using our perturbative QCD approaches, which might be an indication that perturbative QCD or a DGLAP fit to $F_{2}^{D}$ is not valid in this region of phase space without saturation effects [21].

At high $Q^{2}$ (see Fig. 9) the differences between both extrapolations remain small as was the case at medium $Q^{2}$, except at high $x_{\mathbb{P}}$, which is an artefact of the absence of Reggeon contribution in the ZEUS fit.

The fact that no large difference is found between both extrapolations whereas we have large differences in the gluon density shows that we need other measurements more sensitive to the gluon density, like the charm component to $F_{2}^{D}$, or some direct final state studies (jet production, multiplicity studies [23]).

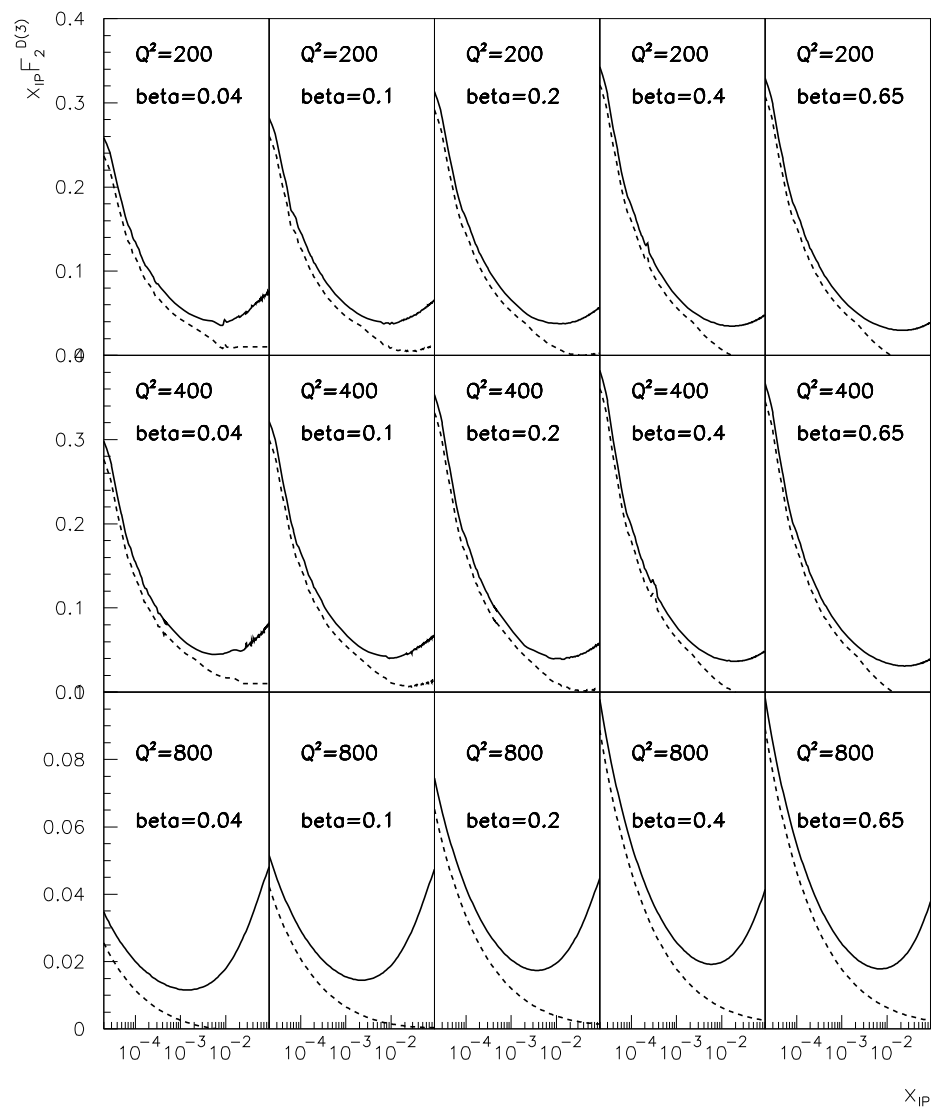

FIG. 9. High $Q^{2}$ extrapolation of the fit results (H1: full line, ZEUS: dashed line). 


\section{Charm contribution and longitudinal diffractive structure functions}

From global QCD fits of the diffractive structure function $F_{2}^{D(3)}$, we have derived reference parton distributions (see Fig. 7). With these distributions, we can now give some predictions for the longitudinal and charm structure functions in diffraction.

We present values for the longitudinal structure function in Fig. 10, which is the sum of two components, namely the perturbative part coming from the QCD fit and the higher-twist component. The higher-twist component of the longitudinal structure function becomes dominant at high value of $\beta$ whereas the low $\beta$ domain is dominated by the perturbative part of $F_{L}$ deduced from QCD fits. We can also evaluate values of $R=\frac{\sigma_{L}}{\sigma_{T}}$ in diffraction, which is illustrated in Fig. 11. Here again, the high $\beta$ domain represents the higher-twist component.

We note that the differences between the H1 and ZEUS fit results are quite small in $F_{L}$. However, a measurement of $F_{L}^{D}$ or $R$ would be quite interesting to constrain the higher twist component at high $\beta$. The $R$ values are more different between $\mathrm{H} 1$ and ZEUS predictions, but the error bars on these extrapolations are quite large.

The charm predictions are shown in Fig. 12, 13. We note the large differences between the ZEUS and H1 fit results which shows the importance of this direct measurement, which is directly sensitive to the gluon density. The charm contribution to $F_{2}^{D}$ is expected to be larger than for the proton structure function (about $40 \%$ at $\beta \sim 0.1, Q^{2} \sim 10$ $\mathrm{GeV}^{2}$ ) if we consider the results of the $\mathrm{H} 1$ fit, whereas the results of the ZEUS fit are more similar to the proton values 10 .

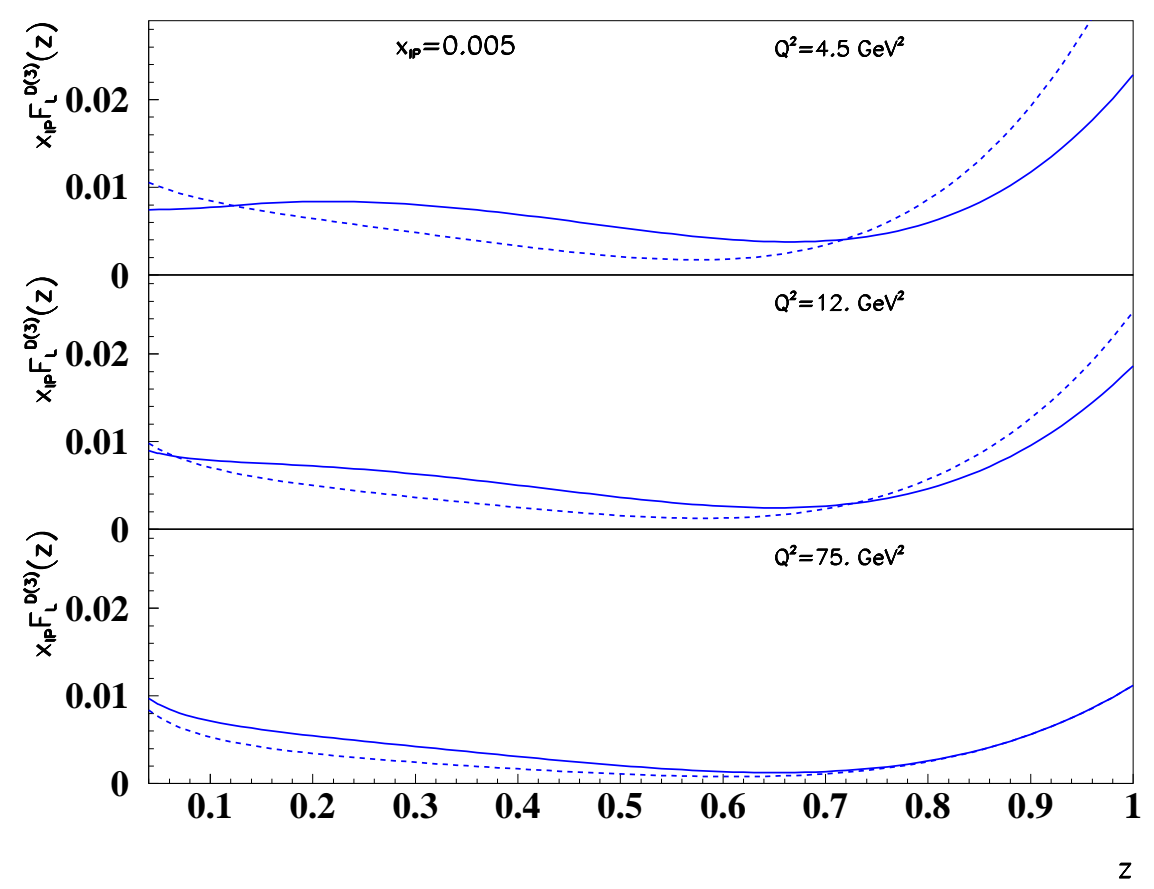

FIG. 10. Prediction for the longitudinal structure function $x_{\mathbb{P}} \cdot F_{L}^{D(3)}$ (at fixed $x_{\mathbb{P}}=0.005$ ) presented as a function of $z=\beta$ for different values of $Q^{2}$ (H1: full line, ZEUS: dashed line).

\footnotetext{
${ }^{10}$ Preliminary H1 and ZEUS measurements [22] show that the H1 (resp. ZEUS) charm cross-section is closer to the results of the predictions coming from the ZEUS (resp. H1) QCD fit. More precise data are needed to solve these discrepencies.
} 


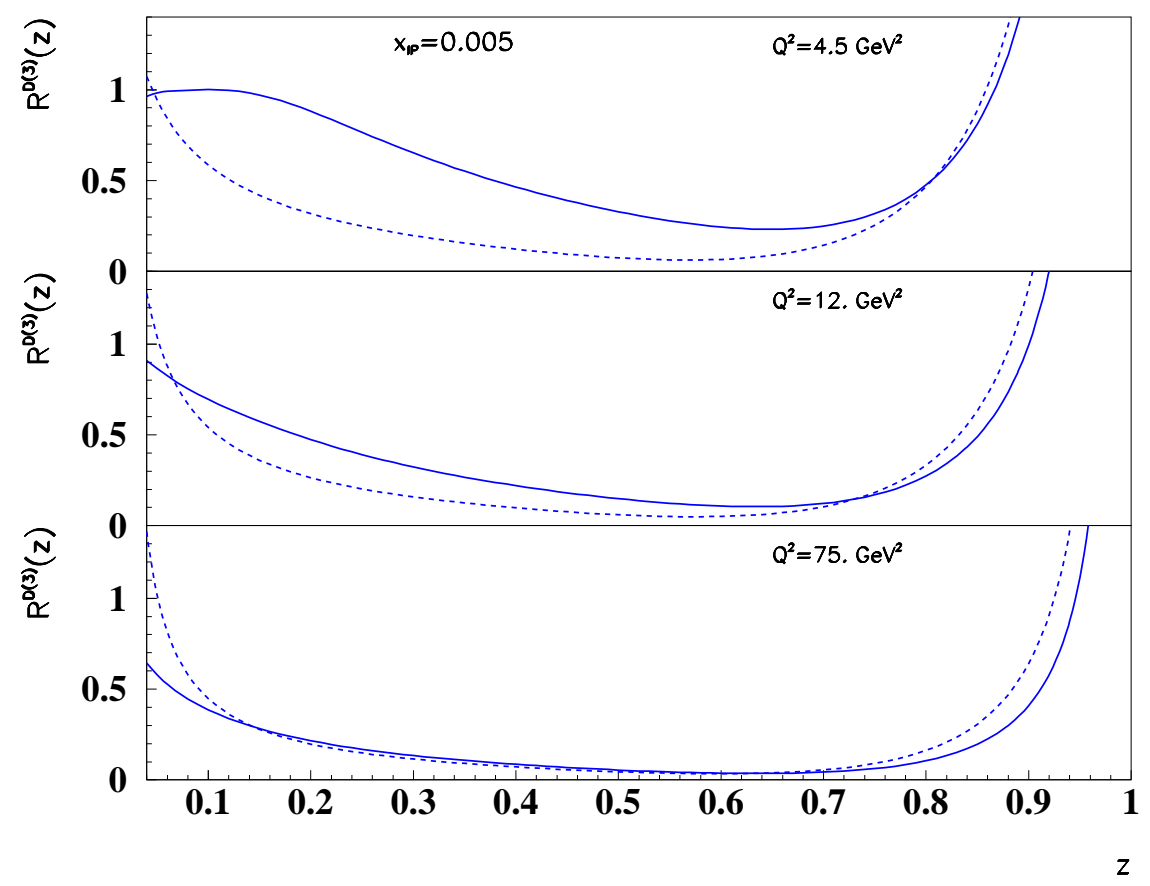

FIG. 11. Prediction for $R^{D(3)}$ presented as a function of $z=\beta$ for different values of $Q^{2}$ (H1: full line, ZEUS: dashed line).

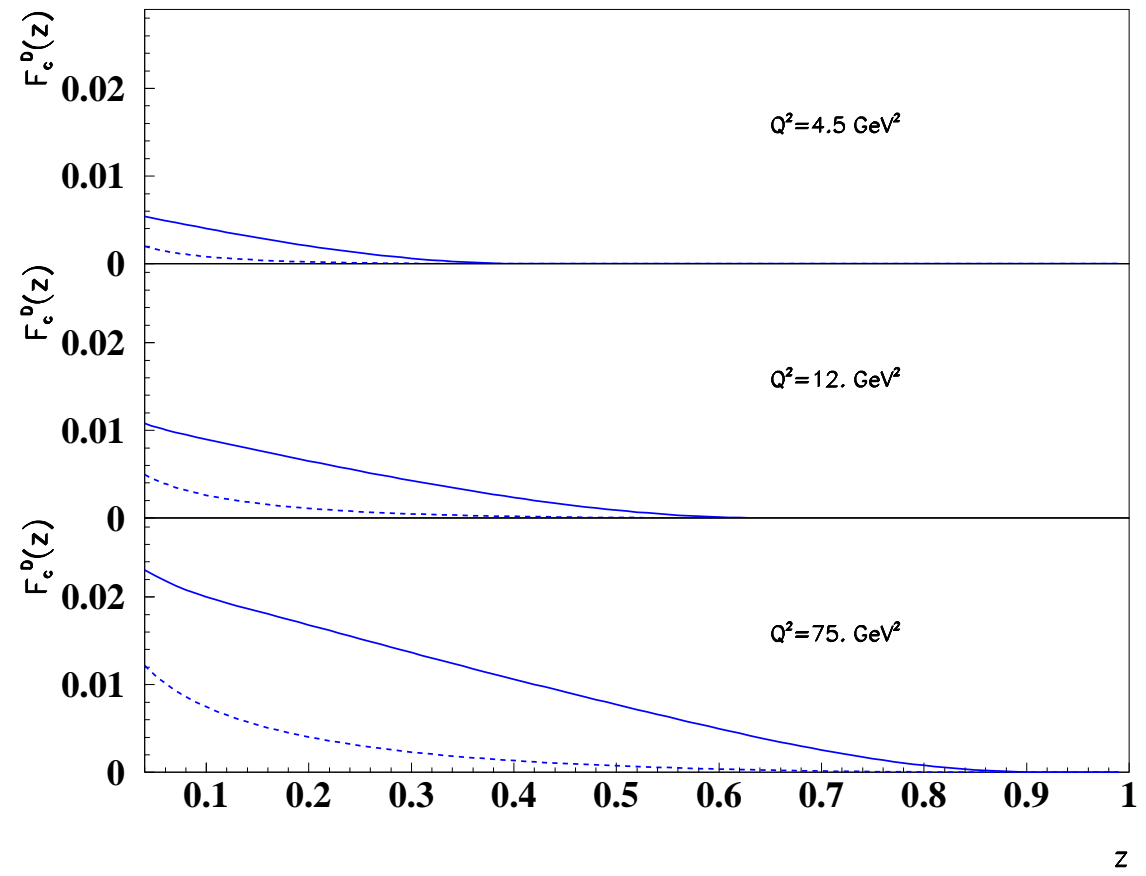

FIG. 12. Prediction for the charm structure function $F_{c}^{D}$ presented as a function of $z=\beta$ for different values of $Q^{2}$ (H1: full line, ZEUS: dashed line). 


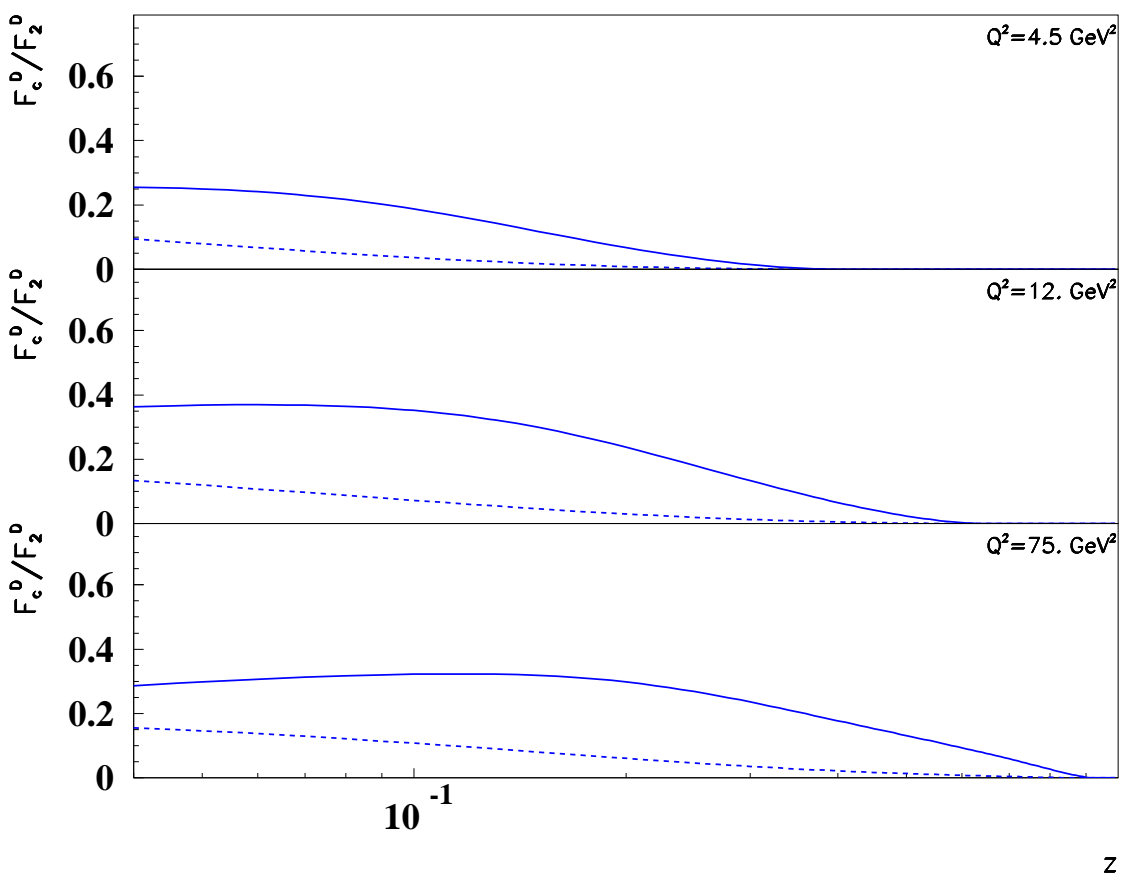

FIG. 13. Prediction for the fraction of charm structure function $F_{2}^{D(c \bar{c})} / F_{2}^{D}$ presented as a function of $z=\beta$ for different values of $Q^{2}$ (H1: full line, ZEUS: dashed line). 


\section{EXTRAPOLATION TO TEVATRON AND COMPARISON WITH CDF DATA}

The QCD fits we obtained from HERA data allow us to make direct comparisons for measurements at the Tevatron. It is quite interesting to be able to directly test factorization breaking between HERA and the Tevatron using the measurements performed at both accelerators. We thus compare the extrapolations of the H1 and ZEUS QCD fits to the recent CDF diffractive jet cross-section measurement [7]. The result is given in Fig. 14. We note a large discrepancy both in shape and normalization between $\mathrm{H} 1$ predictions and CDF data, clearly showing factorization breaking. However, the ZEUS fits are more compatible in normalization with the CDF measurement even if the shape is not described properly. We know that the ZEUS gluon density is between 2 and 3 times smaller than the H1 one. The predictions for the Tevatron are thus expected to be very much different by a factor 2 to 3 between H1 and ZEUS since they correspond to single Pomeron exchange. If the large statistical and systematic uncertainties on the gluon density are taken into account (about $50 \%$ for ZEUS, $25 \%$ for H1), ZEUS data are compatible with factorization at low $\beta$. One should, however, question whether one has the right to extrapolate ZEUS results without introducing an additional Reggeon component. Namely the CDF measurement is in a region in $x_{\mathbb{P}}$ where the Reggeon contribution is important, in contrary to the ZEUS measurement. The error bar on the ZEUS fit extrapolation is thus enhanced due to this uncertainty. Concerning the extrapolation of the $\mathrm{H} 1$ fit and the comparison with CDF data, one must notice that the CDF data lie primilarly at low values of $\beta$ where there are few H1 data points. Moreover, in this kinematical domain, the $\mathrm{H} 1$ data have the tendency to lie more in the high $x_{\mathbb{P}}$ region where the Reggeon contribution is important and not well constrained by the fit. Thus, the extrapolation to the CDF domain suffers from large uncertainties. A combined fit using CDF data to constrain the low $\beta$ region and the HERA data to constrain the high $\beta$ domain would thus be of great interest.

More precise data from HERA and more detailed comparisons with Tevatron are thus needed to study precisely factorization breaking between both experiments. The discussion of eventual higher twist contributions is clearly also valuable in order to reach conclusions on the shape at larger $\beta$. It is thus important to get an accurate measurement of the gluon density in the Pomeron at low values of $\beta$ from HERA. Furthermore the Forward Proton Detector [25] installed by the D0 collaboration for Run II will be of great help to get a direct measurement of the diffractive structure functions. 


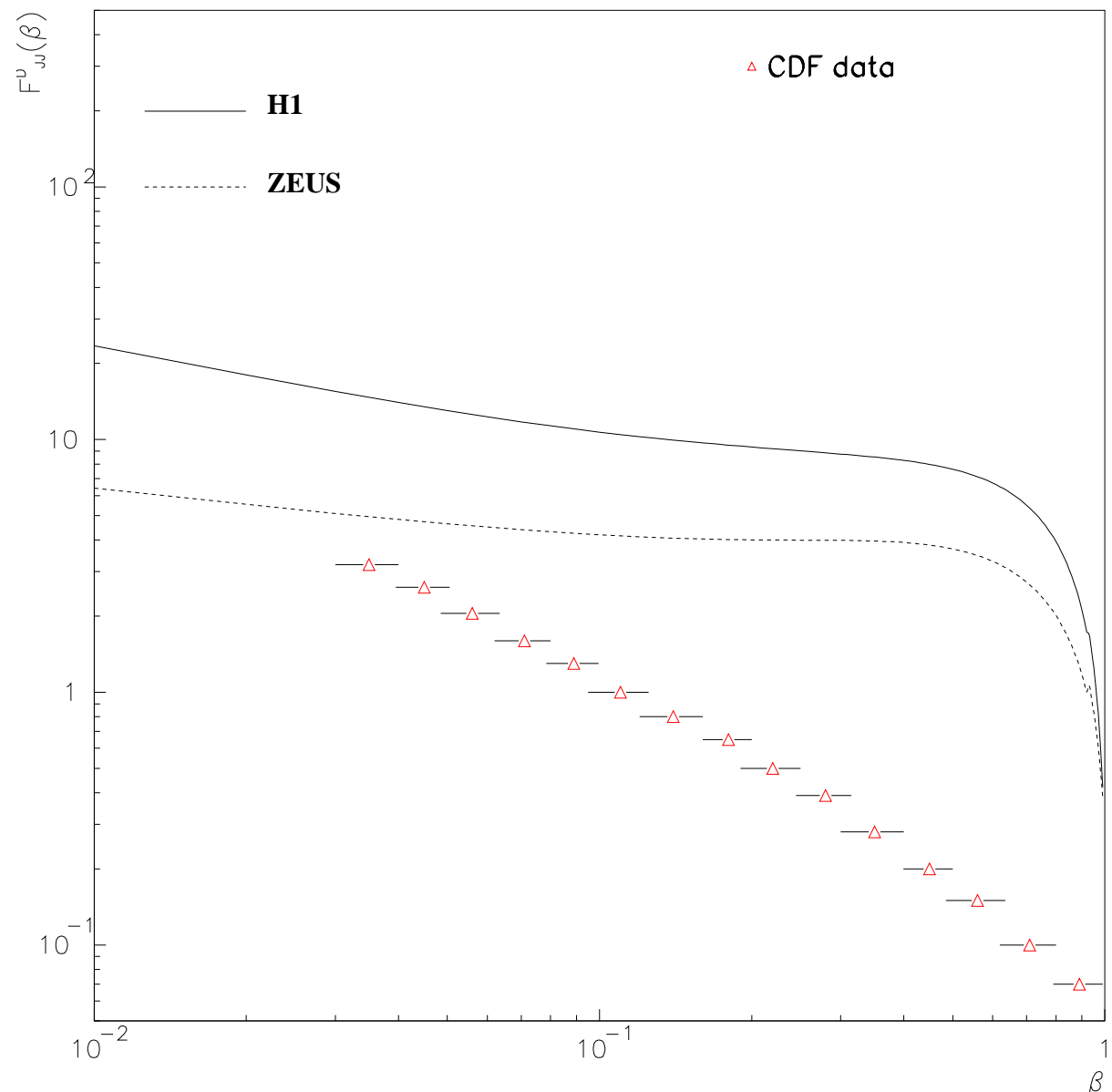

FIG. 14. CDF data $\beta$ distributions compared with extrapolations from the parton densities of the proton extracted from diffractive $F_{2}^{D(3)}$ measurements from $\mathrm{H} 1$ and ZEUS collaborations 


\section{CONCLUSION}

Let us again go through the points discussed in the introduction:

-We have developped a new method to get the Pomeron and Reggeon exponents using Regge fits, confirming a higher value of the Pomeron intercept in "hard" diffraction than in soft processes using a more precise method.

-We propose (and check the stability) of a new NLO DGLAP fit to the diffractive structure functions. We have obtained parton distributions for the Pomeron using $\mathrm{H} 1$ and ZEUS data.

-H1 and ZEUS data both require a large gluon component inside the Pomeron but the gluon densities are quite different. The quark densities are found to be similar in shape and magnitude. Using a parametrisation for the higher twist contribution to $F_{2}^{D}$ allows us to get a description of the full set of data using the NLO DGLAP evolution equations, and leads to the same parton distribution as before. The longitudinal diffractive structure function measurement could allow us to further constrain these higher twist parametrisations.

-Differences in the H1 and ZEUS gluon distributions lead to significant differences in the longitudinal and charm contributions to $F_{2}^{D}$. The low $Q^{2}$ extrapolation shows some scaling violations which do not seem to be present in the data, which might be a sign of saturation effects.

-The same difference leads to diverging extrapolations for single diffraction at the Tevatron. While the H1-based extrapolation leads to a strong factorization breaking in both shape and normalization (at least a factor of 10 with respect to CDF data), the ZEUS-based extrapolation leads to a possible compatibility with factorization at least in the small $\beta$ (large diffractive mass) range.

All these questions deserve a development of experimental and theoretical work in this domain, with the prospects

of undestanding key aspects of QCD properties of "hard" diffraction. The possibility of diffractive production of heavy objects (jets, Higgses) at tevatron even enhances the interest of such studies.

\section{ACKNOWLEDGMENTS}

We thank A.Brandt, D.Goulianos, P.Marage, P.Newman, and P.Van Mechelen for useful discussions and a careful reading of the manuscript. 


\section{Appendix A1: Decorrelation method to get the Pomeron and Reggeon intercepts}

Rewriting equation (1) as

$$
F_{2}^{D(3)}\left(Q_{j}^{2}, \beta_{k}, x_{\mathbb{P}, \mathrm{i}}\right)=f_{\mathbb{P} / \mathrm{p}}\left(x_{\mathbb{P}, \mathrm{i}}\right) A_{j k}\left(Q_{j}^{2}, \beta_{k}\right)+f_{\mathbb{R} / \mathrm{p}}\left(x_{\mathbb{P}, \mathrm{i}}\right) B_{j k}\left(Q_{j}^{2}, \beta_{k}\right)
$$

A fit is then performed in each $\left(Q_{j}^{2}, \beta_{k}\right)$ bins. $A$ and $B$ are only related to the $Q_{j}^{2}, \beta_{k}$ bin considered, which means that they can be fitted within this bin by minimizing the following $\chi_{j k}^{2}\left(Q_{j}^{2}, \beta_{k}\right)$ functions

We have $\chi^{2}=\sum_{j} \sum_{k} \chi_{j k}^{2}\left(Q_{j}^{2}, \beta_{k}\right)$ and

$$
\chi_{j k}^{2}\left(Q_{j}^{2}, \beta_{k}\right)=\sum_{i} \frac{\left(f_{\mathbb{P} / \mathrm{p}}\left(x_{\mathbb{P}, \mathrm{i}}\right) A_{j}\left(Q_{j}^{2}, \beta_{k}\right)+f_{\mathbb{R} / \mathrm{p}}\left(x_{\mathbb{P}, \mathrm{i}}\right) B_{j}\left(Q_{j}^{2}, \beta_{k}\right)-F_{2}^{D(3), \text { exper. }}\left(Q_{j}^{2}, \beta_{k}, x_{\mathbb{P}, \mathrm{i}}\right)\right)^{2}}{\left.\Delta F_{2}^{D(3), \text { exper. }}\left(Q_{j}^{2}, \beta_{k}, x_{\mathbb{P}, \mathrm{i}}\right)\right)^{2}}
$$

where the $i$ index runs over the number of points with different values of $x_{\mathbb{P}}$ in the $Q_{j}^{2}, \beta_{k}$ bin considered and $\Delta F_{2}^{D(3), \text { exper. }}$ is the statistical error on $F_{2}^{D}$ (denoted $\sigma_{i}$ in the following). We use the notations

$$
\begin{aligned}
& f_{\mathbb{P} / \mathrm{p}}\left(x_{\mathbb{P}, \mathrm{i}}\right)=f_{\mathbb{P}, \mathrm{i}} \\
& f_{\mathbb{R} / \mathrm{p}}\left(x_{\mathbb{R}, \mathrm{i}}\right)=f_{\mathbb{R}, \mathrm{i}} \\
& F_{2}^{D(3), \text { exper. }}\left(Q_{j}^{2}, \beta_{k}, x_{\mathbb{P}, \mathrm{i}}\right)=F_{i} .
\end{aligned}
$$

As $A_{j k}$ and $B_{j k}$ do not depend on $x_{\mathbb{P}, \mathrm{i}}$, they can be calculated directly by solving the following system

$$
\begin{aligned}
& \frac{\partial \chi_{j}^{2}\left(Q_{j}^{2}, \beta_{k}\right)}{\partial A_{j k}}=0 \\
& \frac{\partial \chi_{j}^{2}\left(Q_{j}^{2}, \beta_{k}\right)}{\partial B_{j k}}=0
\end{aligned}
$$

which gives

$$
\begin{aligned}
& A_{j k}= \frac{\sum_{i} \frac{2 f_{\mathbb{P}, \mathrm{i}} F_{i}}{\sigma_{i}^{2}} \sum_{i} \frac{2 f_{\mathbb{R}, \mathrm{i}}^{2}}{\sigma_{i}^{2}}-\sum_{i} \frac{2 f_{\mathbb{R}, \mathrm{i}} F_{i}}{\sigma_{i}^{2}} \sum_{i} \frac{2 f_{\mathbb{R}, \mathrm{i}} f_{\mathbb{P}, \mathrm{i}}}{\sigma_{i}^{2}}}{\sum_{i} \frac{2 f_{\mathbb{P}, \mathrm{i}}^{2}}{\sigma_{i}^{2}} \sum_{i} \frac{2 f_{\mathbb{R}, \mathrm{i}}^{2}}{\sigma_{i}^{2}}-\left(\sum_{i} \frac{2 f_{\mathbb{R}, \mathrm{i}} f_{\mathbb{P}, \mathrm{i}}}{\sigma_{i}^{2}}\right)^{2}} \\
& B_{j k}=\frac{\sum_{i} \frac{2 f_{\mathbb{R}, \mathrm{i}} F_{i}}{\sigma_{i}^{2}} \sum_{i} \frac{2 f_{\mathbb{P}, \mathrm{i}}^{2}}{\sigma_{i}^{2}}-\sum_{i} \frac{2 f_{\mathbb{P}, \mathrm{i}} F_{i}}{\sigma_{i}^{2}} \sum_{i} \frac{2 f_{\mathbb{R}, \mathrm{i}} f_{\mathbb{P}, \mathrm{i}}}{\sigma_{i}^{2}}}{\sum_{i} \frac{2 f_{\mathbb{P}, \mathrm{i}}^{2}}{\sigma_{i}^{2}} \sum_{i} \frac{2 f_{\mathbb{R}, \mathrm{i}}^{2}}{\sigma_{i}^{2}}-\left(\sum_{i} \frac{2 f_{\mathbb{R}, \mathrm{i}} f_{\mathbb{P}, \mathrm{i}}}{\sigma_{i}^{2}}\right)^{2}}
\end{aligned}
$$

Thus, for each $Q_{j}^{2}, \beta_{k}$ bin, $A_{j k}$ and $B_{j k}$ are well defined values and depend on $\alpha_{\mathbb{P}}(0)$ and $\alpha_{\mathbb{R}}(0)$ via the flux factors. In conclusion, all $A_{j k}$ and $B_{j k}$ parameters should not be introduced without care as free parameters in the global Regge fit as they satisfy the two last expressions in terms of $F_{i}, f_{\mathbb{P}, \mathrm{i}}$ and $f_{\mathbb{R}, \mathrm{i}}$, which reduces considerably the number of free parameters. Indeed only 2 free parameters remain : $\alpha_{\mathbb{P}}(0)$ and $\alpha_{\mathbb{R}}(0)$. 


\section{Appendix A2: ZEUS and H1 fit parameters}

$F_{2}^{D(3)}$ can be expressed as a sum of two factorized contributions corresponding to a Pomeron and secondary Reggeon trajectories. The Pomeron and Reggeon fluxes are assumed to follow a Regge behaviour with linear trajectories $\alpha_{\mathbb{P}, \mathbb{R}}(t)=\alpha_{\mathbb{P}, \mathbb{R}}(0)+\alpha_{\mathbb{P}, \mathbb{R}}^{\prime} t$, such that

$$
f_{\mathbb{P} / p, \mathbb{R} / p}\left(x_{\mathbb{P}}\right)=\int_{t_{c u t}}^{t_{\text {min }}} \frac{e^{B_{\mathbb{P}, \mathbb{R}} t}}{x_{\mathbb{P}}^{2 \alpha_{\mathbb{P}, \mathbb{R}}(t)-1}} \mathrm{~d} t
$$

where $\left|t_{\text {min }}\right|$ is the minimum kinematically allowed value of $|t|$ and $t_{c u t}=-1 \mathrm{GeV}^{2}$ is the limit of the measurement.

$$
F_{2}^{D(3)}\left(Q^{2}, \beta, x_{\mathbb{P}}\right)=f_{\mathbb{P} / \mathrm{p}}\left(x_{\mathbb{P}}\right) F_{2}^{\mathbb{P}}\left(Q^{2}, \beta\right)+N_{\mathbb{R}} f_{\mathbb{R} / \mathrm{p}}\left(x_{\mathbb{P}}\right) F_{2}^{\mathbb{R}}\left(Q^{2}, \beta\right) .
$$

The values of $\alpha_{\mathbb{P}, \mathbb{R}}(0)$ are free parameters while $B_{\mathbb{P}, \mathbb{R}}$ and $\alpha_{\mathbb{P}, \mathbb{R}}^{\prime}$ are taken from hadron-hadron data $\left(\alpha_{\mathbb{P}}^{\prime}=0.26\right.$ $\mathrm{GeV}^{-2}, \alpha_{\mathbb{R}}^{\prime}=0.90 \mathrm{GeV}^{-2}, B_{\mathbb{P}}=4.6 \mathrm{GeV}^{-2}, B_{\mathbb{P}}=2.0 \mathrm{GeV}^{-2}$ The Pomeron intercept coming from Regge fits is $\alpha_{\mathbb{P}}(0)=1.20 \pm 0.02$ for $\mathrm{H} 1$, and $\alpha_{\mathbb{P}}(0)=1.13 \pm 0.04$ for ZEUS.

The Reggeon structure function is needed only to describe H1 data, and the value of $\alpha_{\mathbb{R}}(0)=0.62 \pm 0.02$, with the normalization $N_{\mathbb{R}}=14.2 \pm 0.1$. The values of $F_{2}^{\mathbb{R}}\left(Q^{2}, \beta\right)$ are taken from a parameterisation of the pion structure function [9].

The parametrisations for the gluon and quark densities $\left(z S_{q}\left(z, Q^{2}\right)=u+\bar{u}+d+\bar{d}+s+\bar{s}\right)$ in the Pomeron are given at $Q_{0}^{2}=3 \mathrm{GeV}^{2}$ :

$$
\begin{aligned}
& z S\left(z, Q^{2}=Q_{0}^{2}\right)=\left[\sum_{j=1}^{n} C_{j}^{(S)} \cdot P_{j}(2 z-1)\right]^{2} \cdot e^{\frac{a}{z-1}} \\
& z G\left(z, Q^{2}=Q_{0}^{2}\right)=\left[\sum_{j=1}^{n} C_{j}^{(G)} \cdot P_{j}(2 z-1)\right]^{2} \cdot e^{\frac{a}{z-1}}
\end{aligned}
$$

where $P_{j}(\zeta)$ is the $j^{\text {th }}$ member in a set of Chebyshev polynomials, which are chosen such that $P_{1}=1, P_{2}=\zeta$ and $P_{j+1}(\zeta)=2 \zeta P_{j}(\zeta)-P_{j-1}(\zeta)$

\begin{tabular}{|c|c|c|}
\hline parameters & H1 & ZEUS \\
\hline \hline$C_{1}^{(S)}$ & $0.18 \pm 0.05$ & $0.41 \pm 0.02$ \\
$C_{2}^{(S)}$ & $0.07 \pm 0.02$ & $-0.16 \pm 0.03$ \\
$C_{3}^{(S)}$ & $-0.13 \pm 0.02$ & $-0.11 \pm 0.02$ \\
\hline$C_{1}^{(G)}$ & $0.82 \pm 0.40$ & $0.53 \pm 0.30$ \\
$C_{2}^{(G)}$ & $0.22 \pm 0.06$ & $0.28 \pm 0.25$ \\
$C_{3}^{(G)}$ & $0.01 \pm 0.04$ & $0.02 \pm 0.11$ \\
\hline
\end{tabular}

Table I- Pomeron quark and gluon densities parameters

The parametrisation for the higher-twist contribution to the diffractive structure function is the following

$$
F_{2}^{D(3), I I I}=C\left(\frac{x_{0}}{x_{\mathbb{P}}}\right)^{n_{4}}\left(\frac{Q_{0}^{2}}{Q^{2}}\right)\left(\ln \left(\frac{Q^{2}}{4 Q_{0}^{2} \beta}+1.75\right)\right)^{2} \beta^{3}(1-2 \beta)^{2}
$$

where

$$
n_{2,4}=n_{4}^{0}+n_{4}^{1} \ln \left[\ln \left(\frac{Q^{2}}{Q_{0}^{2}}\right)+1\right]
$$

with the following values for the parameters 


\begin{tabular}{|c|c|c|}
\hline parameters & H1 & ZEUS \\
\hline \hline$C$ & $0.035 \pm 0.016$ & $357 . \pm 106$. \\
$x_{0}$ & $0.40 \pm 0.02$ & $0.0008 \pm 0.0002$ \\
$n_{4}^{0}$ & $1.43 \pm 0.08$ & $1.00 \pm 0.10$ \\
$n_{4}^{1}$ & $0.00 \pm 0.05$ & $0.37 \pm 0.10$ \\
\hline
\end{tabular}

Table II: Parameters obtained for the higher-twist parametrisation 
[1] H1 Collaboration, Z. Phys. C76 (1997) 613.

[2] ZEUS Collaboration, Eur.Phys.J.C6 (1999) 43.

[3] G. Ingelman, P. Schlein, Phys. Lett. B 152 (1985) 256.

[4] J.Collins, Phys.Rev. D57 (1998) 3051, Erratum-ibid. D61 (2000) 019902, F. Hautmann, Z. Kunszt, D. E. Soper "Diffractive parton distributions in light-cone QCD", hep-ph/9911278.

[5] G.Altarelli and G.Parisi, Nucl. Phys. B126 18C (1977) 298. V.N.Gribov and L.N.Lipatov, Sov. Journ. Nucl. Phys. (1972) 438 and 675. Yu.L.Dokshitzer, Sov. Phys. JETP. 46 (1977) 641.

[6] H. Jung Comput. Phys. Commun. 86 (1995) 147.

[7] CDF Coll., preprint FERMILAB-Pub-/055-E CDF, subm. to Phys.Rev.Lett.

[8] H1 Collab., Z. Phys. C74 (1997) 221.

[9] M. Glück, E. Reya, A. Vogt, Z. Phys. C53 (1992) 651.

[10] J.F. Owens, Phys. Rev. D 30 (1984) 943.

[11] S.Donnachie, P.Landshoff Phys. Lett. B 437 (1998) 408, see also K.Covolan, J.Montanha, K.Goulianos, Phys. Lett. B 389 (1996) 176.

[12] H1 Coll., Eur. Phys. J. C6 (1999) 587.

[13] CTEQ QCD evolution code, http ://www.phys.psu.edu/cteq/

[14] L. Schoeffel, Nucl.Instrum.Meth. A423 (1999) 439.

[15] M. Glück, E. Hoffmann, E. Reya, Z. Phys. C13 (1982) 119., M. Glück, E. Reya, M. Stratmann, Nucl. Phys. B422 (1994) 37.

[16] J.Bartels, J.Ellis, H.Kowalski, M.Wuesthoff, Eur.Phys.J. C7 (1999) 443, J.Bartels, C.Royon, Mod. Phys. Lett. A14 (1999) 1583.

[17] A.Bialas, R.Peschanski, C.Royon, Phys. Rev. D57 (1998) 6899. S.Munier, R.Peschanski, C.Royon, Nucl. Phys. B534 (1998) 297.

[18] L.N.Lipatov, Sov. J. Nucl. Phys. 23 (1976) 642, V.S.Fadin, E.A.Kuraev and L.N.Lipatov, Phys. lett. B60 (1975) 50; E.A.Kuraev, L.N.Lipatov and V.S.Fadin, Sov.Phys.JETP 44 (1976) 45, 45 (1977) 199, I.I.Balitsky and L.N.Lipatov, Sov.J.Nucl.Phys. 28 (1978) 822.

[19] A.H.Mueller and B.Patel, Nucl. Phys. B425 (1994) 471, A.H.Mueller, Nucl. Phys. B437 (1995) 107, A.H.Mueller, Nucl. Phys. B415 (1994) 373; H.Navelet, R.Peschanski, Ch.Royon, S.Wallon, Phys. Lett. B385 (1996) 357, H. Navelet, R. Peschanski, C. Royon. Phys. Lett. B366 (1996)329.

[20] C.Royon, Contribution to the DIS99 workshop for the H1 Collaboration, Zeuthen (Germany), 19-23 April 1999, hepph/9908216, M.Capua, Contribution to the DIS2000 workshop for the ZEUS Collaboration, Liverpool (U.K.), 25-30 April 2000.

[21] K. Golec-Biernat, M. Wüsthoff, Phys.Rev. D60 (1999) 114023.

[22] J.Cole, Contribution to the DIS2000 workshop for the ZEUS Collaboration, Liverpool (U.K.), 25-30 April 2000.

[23] H1 Coll.,Phys. Lett. B428 (1998) 206, Eur. Phys. J. C 5 (1998) 3, ZEUS Coll., Phys. Lett. B 421 (1998) 368.

[24] H1 coll., Nucl.Phys. B470 (1996) 3.

[25] D0 Coll., D0 FPD Proposal, FNAC PAC Meeting October 18, 1997. 